\title{
Evolution of Long Term Variability in Solar Analogs
}

\author{
Ricky Egeland ${ }^{1,2}$, Willie Soon ${ }^{3}$, Sallie Baliunas ${ }^{4}$, Jeffrey C. Hall ${ }^{5}$ and \\ Gregory W. Henry ${ }^{6}$ \\ ${ }^{1}$ High Altitude Observatory/NCAR, 3080 Center Green Dr, Boulder CO, 80301, USA \\ email: egeland@ucar.edu \\ ${ }^{2}$ Dept. of Physics, Montana State University, P.O. Box 173840, Bozeman MT 59717, USA \\ ${ }^{3}$ Harvard-Smithsonian Center for Astrophysics, Cambridge, MA 02138, USA \\ ${ }^{4}$ No affiliation \\ ${ }^{5}$ Lowell Observatory, 1400 West Mars Hill Road, Flagstaff, AZ 86001, USA \\ ${ }^{6}$ Center of Excellence in Information Systems, Tennessee State University, 3500 John A. \\ Merritt Blvd., Box 9501, Nashville, TN 37209, USA
}

\begin{abstract}
Earth is the only planet known to harbor life, therefore we may speculate on how the nature of the Sun-Earth interaction is relevant to life on Earth, and how the behavior of other stars may influence the development of life on their planetary systems. We study the long-term variability of a sample of five solar analog stars using composite chromospheric activity records up to 50 years in length and synoptic visible-band photometry about 20 years long. This sample covers a large range of stellar ages which we use to represent the evolution in activity for solar mass stars. We find that young, fast rotators have an amplitude of variability many times that of the solar cycle, while old, slow rotators have very little variability. We discuss the possible impacts of this variability on young Earth and exoplanet climates.
\end{abstract}

Keywords. Sun Activity Chromosphere Variability Climate Habitability

\section{Introduction}

The cyclic variability of the solar sunspot count was noted by Schwabe (1844), but observations of surface activity in Sun-like stars came more than a century later. Wilson (1968) introduced the Mount Wilson Observatory (MWO) HK Project, which began synoptic monitoring of the emission in the cores of Fraunhofer $\mathrm{H}(3968.47 \AA$ ) and $\mathrm{K}$ (3933.66 ^) K lines for a sample of Sun-like (F, G, and K-type) stars. Formed by singlyionized calcium, these lines have a reversal feature for which the central emission has long been known to correlate with regions of strong magnetic field on the Sun (Leighton 1959; Linsky and Avrett 1970). Using Ca II H \& K as a proxy for magnetic activity on stars, Wilson (1978) presented observations of 91 main-sequence stars, showing that they do in fact vary, and that several of the stars appeared to have completed a cycle in HK flux variations. Baliunas et al. (1995) summarized $\sim 25$ years of synoptic observations for 112 stars and conclusively showed the existence of cyclic variability, as well as other patterns of variability. More than half the sample showed either erratic variability, longterm trends, or flat activity that may be analogous to the solar Maunder Minimum, a long period of subdued solar activity from about 1650-1700 (Eddy 1976).

Wilson (1968) discussed the difficulty of detecting variability in broad-band visible observations, estimating a 0.001 magnitude ( 1 millimagnitude (mmag); $\approx 0.1 \%$ ) change in solar luminosity due to the passage of spots covering about 1400 millionths of the solar surface. This is comparable to the later measurements of the average cyclic variation in the total solar irradiance (TSI) from the Solar Maximum Mission (Willson and Hudson 
1991). The challenge of measuring visible-band variability in Sun like (FGK-type) stars was taken up by researchers at Lowell Observatory, who used differential photometry of the Strömgren $b$ and $y$ bands to achieve the required precision (Lockwood et al. 1997). They found short-term (inter-year) and long-term (year-to-year) rms amplitudes ranging from $0.002 \mathrm{mag}(0.2 \%)$ to $0.07 \mathrm{mag}(7 \%)$ for about 41 program stars. Overlap with MWO targets allowed the comparison of photometric variability in the combined bandpass $((b+y) / 2)$ to $\mathrm{Ca}$ II $\mathrm{H} \& \mathrm{~K}$ activity expressed with the $R_{\mathrm{HK}}^{\prime}$ index, the ratio of HK flux to the bolometric luminosity. Lockwood et al. (1997) generally found that active stars (high $R_{\mathrm{HK}}^{\prime}$ ) have larger rms photometric variability, and Radick et al. (1998) found a power law relationship between the two quantities. Furthermore, Radick et al. (1998) found that stars were either faculae-dominated, with a positive correlation between brightness and $\mathrm{H} \& \mathrm{~K}$ activity, or spot-dominated, with a negative correlation. The terminology here refers to the dominant features contributing to visible-band brightness variations. The faculae are the photospheric counterpart to the plage in the chromosphere, which are bright features in $\mathrm{Ca}$ II $\mathrm{H} \& \mathrm{~K}$, while spots are dark features in both $\mathrm{H} \& \mathrm{~K}$ emission and visible bandpasses.

Stars like the Sun emit most of their flux in the visible spectrum, and for a planet with an atmosphere like the Earth's, the majority of the radiant energy reaching the surface likewise comes in the visible. The $\sim 0.1 \%$ variability in TSI from the present day Sun is thought to be of little consequence to the globally averaged Earth temperature (Stocker et al. 2013), however this may not have always been the case. The climate impact of the Maunder Minimum period, and its coincidence with the Medieval Little Ice Age are actively debated, however interpretations are crucially dependent on the use of proxy records to extrapolate the present TSI into the Maunder Minimum period (e.g. Kopp 2014; Solanki et al. 2013). The stellar studies of Radick et al. (1998) and Lockwood et al. (2007) show a clear relationship between visible band variability and Ca II H \& K activity, and furthermore it has long been known that stellar activity decreases with age as a star loses angular momentum (Skumanich 1972; Noyes et al. 1984; Barnes 2007). We therefore ask the question, "how has solar variability impacted Earth's climate on stellar evolution (billion year) timescales?", and the related question, "how might stellar variability affect exoplanet climates?"

Clearly the most important impact of stellar evolution on planetary climate is the total flux reaching the top of the atmosphere. According to standard solar evolution models, the luminosity of the Sun has been steadily increasing from an initial value of $\sim 70 \%$ the present-day luminosity when hydrogen burning began $\sim 4.6$ billion years ago (e.g. Gough 1981). Because of the lower luminosity, from first-order calculations we would expect the mean temperature of the Earth to be below the freezing point of water, which is in contradiction to geological evidence for wet conditions and the development of life on Earth 3-4 billion years ago (Sagan and Mullen 1972). This problem is known as the "Faint Young Sun Paradox," which was discussed by Dr. Martens at this symposium. In this contribution, we shall ignore the mean luminosity and consider the climate impact of decadal scale variability from younger, more active stars.

\section{Long-term Variability of Solar Analogs}

To begin to address the questions of the relationship between stellar variability and planetary climate, we look at a sample of five solar-analog stars that may represent the behavior of the Sun at various points in the history of the solar system, as shown in Table 1. This sample is drawn from a larger sample of 26 solar-analog stars with Ca II $\mathrm{H} \& \mathrm{~K}$ observational records up to 50 years in length. These long records are obtained 
Table 1. Stellar Properties \& Variability

\begin{tabular}{lcccccc}
\hline Quantity & HD 20630 & HD 30495 & HD 76151 & HD 146233 & Sun & HD 9562 \\
\hline$M_{V}$ & 5.04 & 4.87 & 4.81 & 4.79 & 4.82 & 3.41 \\
$T / \mathcal{T}_{\odot}^{\mathrm{N}}$ & 0.99 & 1.00 & 0.98 & 1.00 & 1 & 1.01 \\
$R / \mathcal{R}_{\odot}^{\mathrm{N}}$ & 0.93 & 0.97 & 1.05 & 1.02 & 1 & 1.85 \\
$L / \mathcal{L}_{\odot}^{\mathrm{N}}$ & 0.83 & 0.95 & 1.03 & 1.03 & 1 & 3.62 \\
{$[\mathrm{Fe} / \mathrm{H}]$} & 0.00 & -0.08 & -0.04 & -0.02 & 0 & +0.13 \\
$P_{\text {rot }}[\mathrm{d}]$ & 9.2 & 11.36 & 15.0 & 22.7 & 26.09 & 29.0 \\
Age $[\mathrm{Gyr}]$ & $0.5 \pm 0.1$ & $1.0 \pm 0.1$ & $1.4 \pm 0.2$ & $3.66_{-0.50}^{+0.44}$ & 4.57 & $3.4_{-0.2}^{+1.7}$ \\
\hline$\widehat{S}$ & 0.3606 & 0.3020 & 0.2363 & 0.1703 & 0.1686 & 0.1369 \\
$A_{S, 98 \%}$ & 0.1169 & 0.0708 & 0.0679 & 0.0414 & 0.0275 & 0.0226 \\
$A_{S, s}$ & 0.0902 & 0.0502 & 0.0576 & 0.0313 & 0.0203 & 0.0159 \\
$A_{b y, s}[\mathrm{mmag}]$ & 30.1 & 21.5 & 7.9 & 1.3 & $1.5^{\star}$ & 1.8 \\
\hline
\end{tabular}

Notes: Stellar $M_{V}, T_{\text {eff }}$, and $[\mathrm{Fe} / \mathrm{H}]$ are from the Geneva-Copenhagen survey (Holmberg et al. 2009). Stellar luminosities are derived using the empirical bolometric correction of (Flower 1996), and radii follow from the Stephan-Boltzmann Law. $T, R$, and $L$ are given in solar units using the IAU 2015 resolution B2 nominal values (Prša et al. 2016) and have an uncertainty of 1$2 \%$. Rotation periods are from (in order) Gaidos et al. (2000), Egeland et al. (2015) (E15), Donahue et al. (1997), Petit et al. (2008), Donahue et al. (1996), Baliunas et al. (1996). Ages are from (in order) Barnes (2007) (B07), E15, B07, Li et al. (2012), Bouvier (2008), Holmberg et al. (2009). The solar $(b+y) / 2$ amplitude is estimated in Lockwood et al. (2007) by applying a scaling factor to the TSI record.

by combining observations from the MWO HK Project (1966-2003) and the Lowell Observatory SSS (1994-present). Some initial results from this study were presented in Egeland et al. (2016) and Egeland et al. (2016). A similarly long Sun-as-a-star Ca II H \& $\mathrm{K}$ record was developed in Egeland et al. (2017), which accurately placed the long NSO Sacramento Peak Ca II K-line record on the $S$-index scale using coincident observations of the Moon from the MWO HKP-2 instrument. Figure 1 shows the solar $S$-index record and three other stars from our sample on the same scale, illustrating the range of mean activity levels and amplitudes. The youngest, most active star in our sample is HD 20630 $\left(\kappa^{1}\right.$ Ceti), which was discussed at this symposium by Dr. Dias do Nascimento, Jr. Not shown are HD 30495 and HD 146233 (18 Sco), the former which is studied in detail in Egeland et al. (2015), and the latter which is a solar twin (Porto de Mello and da Silva 1997; Meléndez et al. 2014) and has a mean activity and amplitude very similar to the Sun (Hall et al. 2007; Egeland et al. 2017).

Table 1 shows the properties of the sample. All the stars are within $2 \%$ of the solar effective temperature. All but HD 9562 lie very near to the $1 M_{\odot}$ evolutionary track, and therefore approximate the Sun at different points in its lifetime from an age of $0.5 \mathrm{Gyr}$ to the present Sun. HD 9562 is a subgiant which has cooled into the temperature range of our "solar analog" definition, and is more massive than the Sun and the other stars in the sample. Using $\log g=3.99 \pm 0.01$ from Lee et al. (2011) and the radius from Table 1 we obtain $M / \mathcal{M}_{\odot}^{\mathrm{N}}=1.24 \pm 0.05$. Its slow rotation and increased radius are representative of a future Sun, however the Sun is expected to have a lower surface temperature when it similarly expands (see Bressan et al. 2012).

We have computed two estimates of the amplitude of variability in the $S$-index records of this sample. The first is the inter- $98 \%$ range, $A_{S, 98 \%}$, taken as the difference between the top and bottom $1 \%$ of the $\sim 50$ year time series. The thin bars in Figure 1 demonstrate this estimate of amplitude. We also computed full range of the timeseries of seasonal median activity, $A_{S, s}$. Both of these measures of amplitude increase monotonically with 


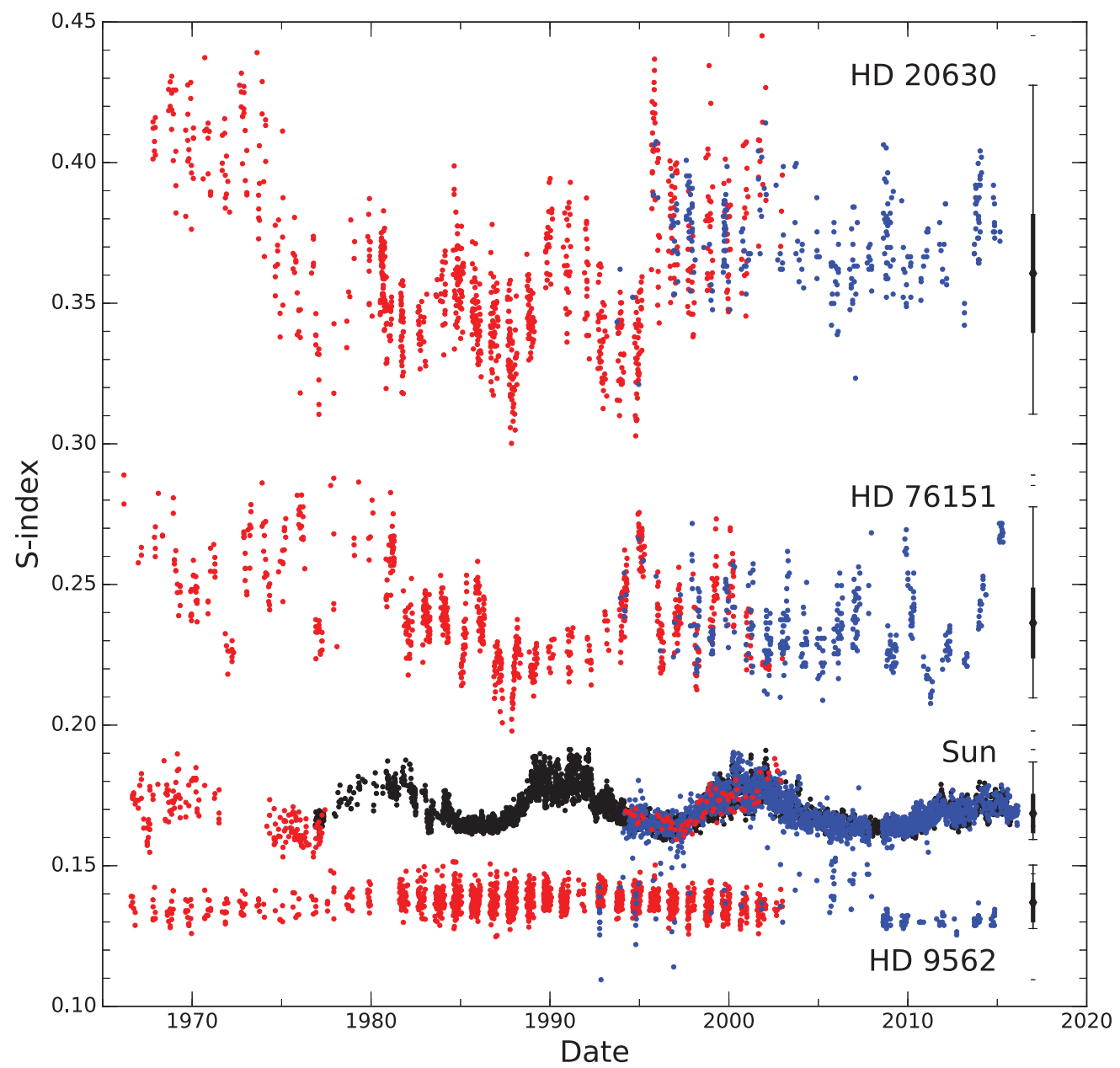

Figure 1. Calibrated composite MWO (red) + SSS (blue) time series for the Sun and three solar analogs. The relative offsets of each time series are real. Data from the Sun are those described in Egeland et al. (2017). The black bar symbol on the right of each time series indicates four quantities: (1) the middle diamond is at the median $S$ for the complete time series, (2) the thin capped bar indicates the location of the 1st and 99th percentile of the data (3) the small dashes indicate the minimum and maximum points and (4) the thick bar is the median seasonal inner-98\% amplitude.

median activity, $\widehat{S}$, but decrease with rotation period, $P_{\text {rot }}$. In fact, from the larger sample of 26 stars we find good linear relationships between median activity and the amplitude, while the relationship with rotation period has significant scatter (Egeland et al. 2017, in preparation). On the Sun, the $S$-index is a proxy for surface magnetic flux (e.g. Harvey and White 1999; Pevtsov et al. 2016). From Table 1 we conclude that the younger Sun had not only higher mean levels of surface flux, but also significantly larger variation in surface flux over decadal timescales. The most active star in our sample, HD $20630\left(\kappa^{1}\right.$ Ceti), has an amplitude of $S$-index variability over four times the solar amplitude. The variability is quite erratic, as can be seen in Figure 1, but a period of reduced activity persists for about two decades from 1975 to 1995 . HD 30495 varies by 2.5 times the solar amplitude, though it appears to have a semi-regular cycle with a period of $\sim 12 \mathrm{yr}$ (Egeland et al. 2015). HD 76151 is also varying with about 2.5 times the solar 


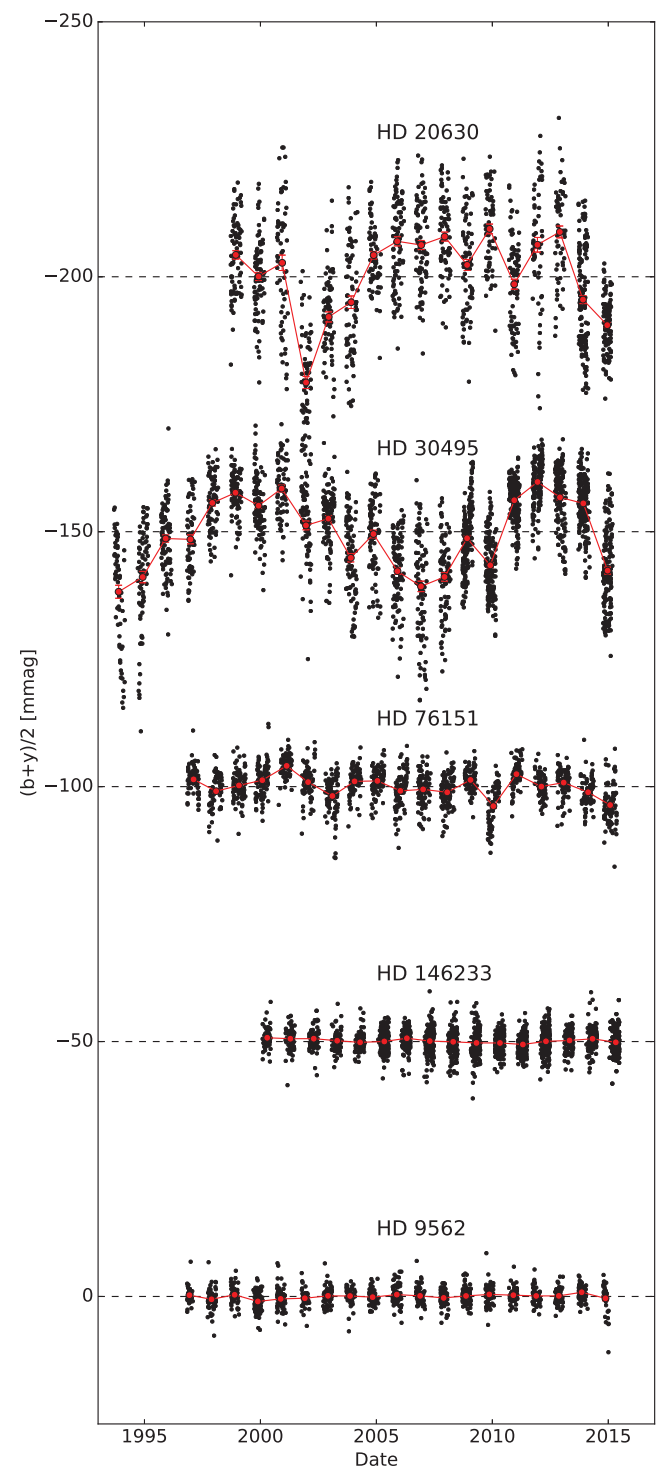

(a)
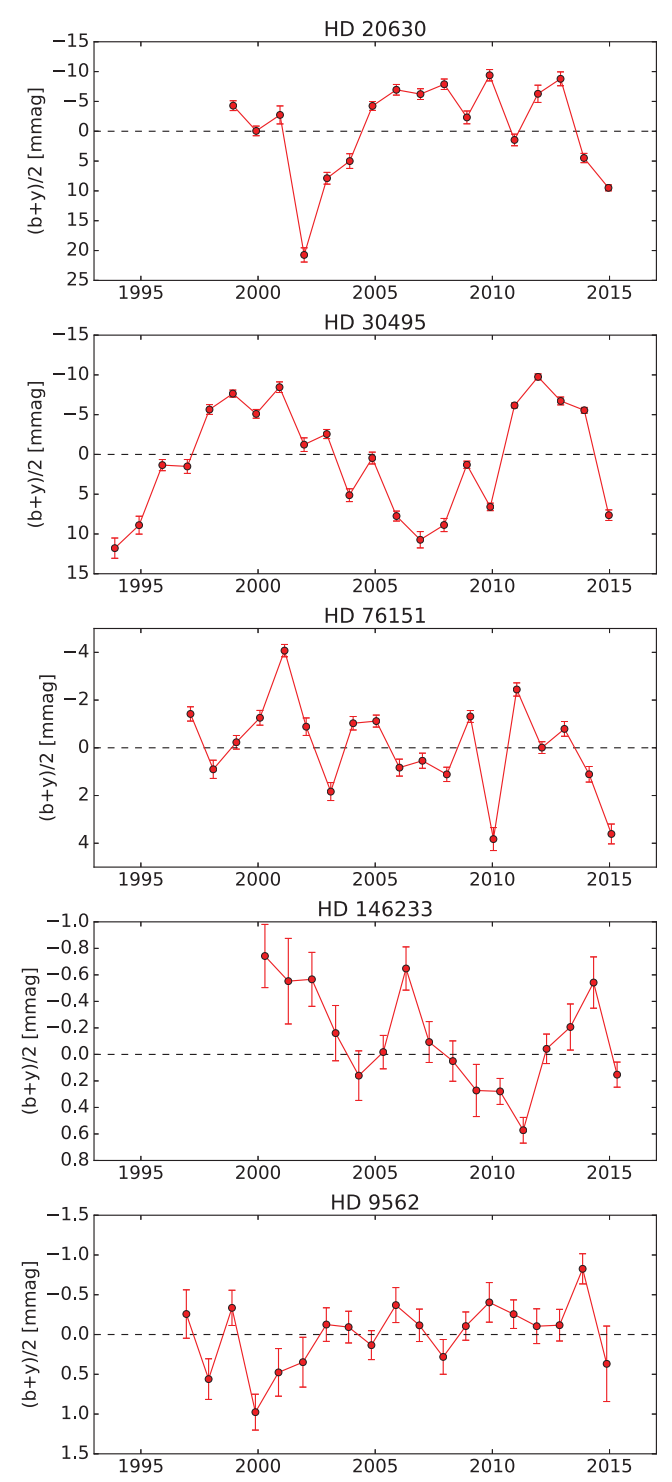

(b)

Figure 2. Variability in the combined Strömgren $(b+y) / 2$ bandpass from the Fairborn Observatory APT differential photometry. Note that the y-axis is reversed so that higher points represent higher brightness. Panel (a) shows the nightly measurements for each star on the same scale, with the arbitrary mean value shifted in increments of 50 mmag. Seasonal means are shown with red points, and are shown again with a smaller scale in panel (b). Error bars indicate the uncertainty of the seasonal mean.

amplitude, but quite erratically. HD 146233 (18 Sco) varies with a 50\% larger amplitude than the Sun in a cyclic fashion (Hall et al. 2007), while the subgiant HD 9562 has an amplitude about $20 \%$ smaller.

Figure 2 shows the variability of our sample in Strömgren $(b+y) / 2$ from the Fairborn Observatory Automated Photometric Telescopes (APT; Henry et al. 1995). The photometric brightness is measured in millimagnitudes (mmag). In these visible bandpasses 
the range of variability across the sample is even more pronounced. The full range of the seasonal means, $A_{b y, s}$, is shown in Table 1 . The Sun's variability in $b+y$ is not well known, but Lockwood et al. (2007) estimate it from the TSI variations and a blackbody approximation of spectral irradiance. $\dagger$ Note that the solar twin 18 Sco has a similar amplitude of variability to the solar estimate. We find that for HD 20630 the amplitude of variability in the visible varies by about twenty times the estimated solar value. HD 30495 varies by about 14 times the solar amplitude, and HD 76151 about 5 times. The flat-activity subgiant 9562 varies slightly more in the visible than the Sun and 18 Sco.

\section{Consequences for Planetary Climate}

What would be the impact on Earth climate if Sun were to vary by twenty times its present value in the visible, as does the young solar analog HD 20630 ( $\kappa^{1}$ Ceti)? If HD 20630 represents the Sun at an age of $\sim 500 \mathrm{Myr}$, then this greatly enhanced variability took place $\sim 4.1$ Gya, at a time when life may have been forming on Earth (Bell et al. 2015). Did the enhanced variability play a role in the development of life on Earth? Is such stellar variability a significant factor in determining the habitability of exoplanets?

To begin to address these questions, we consider the Earth climate study of Meehl et al. (2013), who asked whether a future Maunder Minimum-type event might significantly slow global warming. Meehl et al. (2013) used the Whole Atmosphere Community Climate Model (WACCM) and modified the solar TSI input to include a step-function $0.25 \%$ decrease lasting 50 years. The model produced an immediate response in globally averaged temperature to this small decrease in TSI compared to the baseline case with no prolonged TSI decrease, reducing global temperature by several tenths of a degree centigrade. However, following the period of decreased TSI the warming trend resumed and caught up with the baseline case. Thus, Meehl et al. (2013) concludes that a future Maunder Minimum-like event could slow down, but not stop the global warming trend.

For our purposes, the significant point is that the global temperature registered an immediate response to the small $0.25 \%$ decrease in TSI. When the Sun was like HD 20630 , it may have produced a much larger variations of the order $1-2 \%$. What would solar variability such as this entail for the primitive Earth surface atmosphere and oceans, which were significantly different not only in composition (much less oxygen), but in structure (continental shifts)? More detailed theoretical work is required to determine the importance of such enhanced stellar variability on ancient Earth and exoplanet climate.

R.E. thanks the organizers for the invitation to this symposium and the travel funding provided by the AAS-SPD Thomas Metcalf award. R.E. is supported by the Newkirk Fellowship at the NCAR High Altitude Observatory.

\section{References}

Baliunas, S. L., Donahue, R. A., Soon, W. H., Horne, J. H., Frazer, J., Woodard-Eklund, L., Bradford, M., Rao, L. M., Wilson, O. C., Zhang, Q., Bennett, W., Briggs, J., Carroll, S. M., Duncan, D. K., Figueroa, D., Lanning, H. H., Misch, T., Mueller, J., Noyes, R. W., Poppe,

$\dagger$ The SORCE SIM instrument measured a negative correlation between solar activity and irradiance from 400-691 nm, which covers the $b$ and $y$ bands (Harder et al. 2009). This surprising result remains controversial (e.g. Yeo et al. 2014), and new observations will be required to settle the matter. Judge and Egeland (2015) proposes to place well-characterized reflector into geosynchronous orbit from which a very long, stable timeseries of spectral irradiance using differential photometry could be obtained. 
D., Porter, A. C., Robinson, C. R., Russell, J., Shelton, J. C., Soyumer, T., Vaughan, A. H., \& Whitney, J. H. Chromospheric variations in main-sequence stars. ApJ, 438: 269-287, Jan. 1995. doi:10.1086/175072.

Baliunas, S. L., Nesme-Ribes, E. Sokoloff, D., \& Soon, W. H. A Dynamo Interpretation of Stellar Activity Cycles. ApJ, 460: 848, Apr. 1996. doi:10.1086/177014.

Barnes, S. A. Ages for Illustrative Field Stars Using Gyrochronology: Viability, Limitations, and Errors. ApJ, 669: 1167-1189, Nov. 2007. doi:10.1086/519295.

Bell, E. A., Boehnke, P., Harrison, T. M., \& Mao, W. L. Potentially biogenic carbon preserved in a 4.1 billion-year-old zircon. Proceedings of the National Academy of Sciences, 112 (47): 14518-14521, 2015. doi:10.1073/pnas.1517557112.

Bouvier, J. Lithium depletion and the rotational history of exoplanet host stars. $A \mathscr{E} A, 489$ : L53-L56, Oct. 2008. doi:10.1051/0004-6361:200810574.

Bressan, A., Marigo, P., Girardi, L., Salasnich, B., Dal Cero, C., Rubele, S., \& Nanni, A. PARSEC: stellar tracks and isochrones with the PAdova and TRieste Stellar Evolution Code. MNRAS, 427: 127-145, Nov. 2012. doi:10.1111/j.1365-2966.2012.21948.x.

Donahue, R. A., Saar, S. H., \& Baliunas, S. L. A Relationship between Mean Rotation Period in Lower Main-Sequence Stars and Its Observed Range. ApJ, 466: 384, July 1996. doi:10.1086/177517.

Donahue, R. A., Dobson, A. K., \& Baliunas, S. L. Stellar Active Region Evolution - II. Identification and Evolution of Variance Morphologies in CA II H+K Time Series. Sol. Phys., 171: 211-220, Mar. 1997. doi:10.1023/A:1004922323928.

Eddy, J. A. The Maunder Minimum. Science, 192: 1189-1202, June 1976. doi:10.1126/science.192.4245.1189.

Egeland, R., Metcalfe, T. S., Hall, J. C., \& Henry, G. W. Sun-like Magnetic Cycles in the Rapidly-rotating Young Solar Analog HD 30495. ApJ, 812: 12, Oct. 2015. doi:10.1088/0004$637 \mathrm{X} / 812 / 1 / 12$.

Egeland, R., Soon, W., Baliunas, S., Hall, J. C., Pevtsov, A. A., \& Henry, G. W. Dynamo Sensitivity in Solar Analogs with 50 Years of Ca II H \& K Activity. In G. A. Feiden, editor, Proceedings of the 19th Cambridge Workshop on Cool Stars, Stellar Systems, and the Sun. Zenodo, Sept. 2016. doi:10.5281/zenodo.154118.

Egeland, R., Soon, W., Baliunas, S., Hall, J. C., Pevtsov, A. A., \& Henry, G. W. The solar dynamo zoo. In The 19th Cambridge Workshop on Cool Stars, Stellar Systems, and the Sun. Zenodo, 2016. doi:10.5281/zenodo.57920. URL https://doi.org/10.5281/zenodo. 57920.

Egeland, R., Soon, W., Baliunas, S., Hall, J. C., Pevtsov, A. A., \& Bertello, L. The Mount Wilson Observatory S-index of the Sun. ApJ, 835 (1), January 2017. doi:10.3847/1538$4357 / 835 / 1 / 25$.

Flower, P. J. Transformations from Theoretical Hertzsprung-Russell Diagrams to ColorMagnitude Diagrams: Effective Temperatures, B-V Colors, and Bolometric Corrections. ApJ, 469: 355, Sept. 1996. doi:10.1086/177785.

Gaidos, E. J., Henry, G. W., \& Henry, S. M. Spectroscopy and Photometry of Nearby Young Solar Analogs. AJ, 120: 1006-1013, Aug. 2000. doi:10.1086/301488.

Gough, D. O. Solar interior structure and luminosity variations. Sol. Phys., 74: 21-34, Nov. 1981. doi:10.1007/BF00151270.

Hall, J. C., Lockwood, G. W., \& Skiff, B. A. The Activity and Variability of the Sun and Sun-like Stars. I. Synoptic Ca II H and K Observations. AJ, 133: 862-881, Mar. 2007. doi:10.1086/510356.

Harder, J. W., Fontenla, J. M., Pilewskie, P., Richard, E. C., \& Woods, T. N. Trends in solar spectral irradiance variability in the visible and infrared. Geophys. Res. Lett., 36: L07801, Apr. 2009. doi:10.1029/2008GL036797.

Harvey, K. L. \& White, O. R. Magnetic and Radiative Variability of Solar Surface Structures. I. Image Decomposition and Magnetic-Intensity Mapping. ApJ, 515: 812-831, Apr. 1999. doi:10.1086/307035.

Henry, G. W., Fekel, F. C., \& Hall, D. S. An Automated Search for Variability in Chromospherically Active Stars. AJ, 110: 2926, Dec. 1995. doi:10.1086/117740.

Holmberg, J., Nordström, B., \& Andersen, J. The Geneva-Copenhagen survey of the solar neighbourhood. III. Improved distances, ages, and kinematics. A\&A, 501: 941-947, July 2009. doi:10.1051/0004-6361/200811191. 
Judge, P. G. \& Egeland, R. Century-long monitoring of solar irradiance and Earth's albedo using a stable scattering target in space. MNRAS, 448: L90-L93, Mar. 2015. doi:10.1093/mnrasl/slv004.

Kopp, G. An assessment of the solar irradiance record for climate studies. Journal of Space Weather and Space Climate, 4 (27): A14, Apr. 2014. doi:10.1051/swsc/2014012.

Lee, Y. S., Beers, T. C., Allende Prieto, C., Lai, D. K., Rockosi, C. M., Morrison, H. L., Johnson, J. A., An, D., Sivarani, T., \& Yanny, B. The SEGUE Stellar Parameter Pipeline. V. Estimation of Alpha-element Abundance Ratios from Low-resolution SDSS/SEGUE Stellar Spectra. AJ, 141: 90, Mar. 2011. doi:10.1088/0004-6256/141/3/90.

Leighton, R. B. Observations of Solar Magnetic Fields in Plage Regions. ApJ, 130: 366, Sept. 1959. doi:10.1086/146727.

Li, T. D., Bi, S. L., Liu, K., Tian, Z. J., \& Shuai, G. Z. Stellar parameters and seismological analysis of the star 18 Scorpii. A\&AA, 546: A83, Oct. 2012. doi:10.1051/0004-6361/201219063.

Linsky, J. L. \& Avrett, E. H. The Solar H and K Lines. PASP, 82: 169, Apr. 1970. doi:10.1086/128904.

Lockwood, G. W., Skiff, B. A., \& Radick, R. R. The Photometric Variability of Sun-like Stars: Observations and Results, 1984-1995. ApJ, 485: 789-811, Aug. 1997.

Lockwood, G. W., Skiff, B. A., Henry, G. W., Henry, S., Radick, R. R., Baliunas, S. L., Donahue, R. A., \& Soon, W. Patterns of Photometric and Chromospheric Variation among Sun-like Stars: A 20 Year Perspective. ApJS, 171: 260-303, July 2007. doi:10.1086/516752.

Meehl, G. A., Arblaster, J. M., \& Marsh, D. R. Could a future "Grand Solar Minimum" like the Maunder Minimum stop global warming? Geophys. Res. Lett., 40: 1789-1793, May 2013. doi:10.1002/grl.50361.

Meléndez, J., Ramírez, I., Karakas, A. I., Yong, D., Monroe, T. R. Bedell, M., Bergemann, M., Asplund, M., Tucci Maia, M., Bean, J., do Nascimento, Jr., J.-D., Bazot, M., AlvesBrito, A., Freitas, F. C., \& Castro, M. 18 Sco: A Solar Twin Rich in Refractory and Neutron-capture Elements. Implications for Chemical Tagging. ApJ, 791: 14, Aug. 2014. doi:10.1088/0004-637X/791/1/14.

Noyes, R. W., Hartmann, L. W., Baliunas, S. L., Duncan, D. K., \& Vaughan, A. H. Rotation, convection, and magnetic activity in lower main-sequence stars. ApJ, 279: 763-777, Apr. 1984. doi:10.1086/161945.

Petit, P., Dintrans, B., Solanki, S. K., Donati, J.-F., Aurière, M., Lignières, F., Morin, J., Paletou, F., Ramirez Velez, J., Catala, C., \& Fares, R. Toroidal versus poloidal magnetic fields in Sun-like stars: a rotation threshold. MNRAS, 388: 80-88, July 2008. doi:10.1111/j.13652966.2008.13411.x.

Pevtsov, A. A., Virtanen, I., Mursula, K., Tlatov, A., \& Bertello, L. Reconstructing solar magnetic fields from historical observations. I. Renormalized Ca K spectroheliograms and pseudo-magnetograms. A\&A, 585: A40, Jan. 2016. doi:10.1051/0004-6361/201526620.

Porto de Mello, G. F. \& da Silva, L. HR 6060: The Closest Ever Solar Twin? ApJ, 482: L89, June 1997. doi:10.1086/310693.

Prša, A., Harmanec, P., Torres, G., Mamajek, E., Asplund, M., Capitaine, N., ChristensenDalsgaard, J., Depagne, É., Haberreiter, M., Hekker, S., Hilton, J., Kopp, G., Kostov, V., Kurtz, D. W. Laskar, J., Mason, B. D., Milone, E. F., Montgomery, M., Richards, M., Schmutz, W., Schou, J., \& Stewart, S. G. Nominal Values for Selected Solar and Planetary Quantities: IAU 2015 Resolution B3. AJ, 152: 41, Aug. 2016. doi:10.3847/0004$6256 / 152 / 2 / 41$.

Radick, R. R., Lockwood, G. W., Skiff, B. A., \& Baliunas, S. L. Patterns of Variation among Sun-like Stars. ApJS, 118: 239-258, Sept. 1998. doi:10.1086/313135.

Sagan, C. \& Mullen, G. Earth and Mars: Evolution of Atmospheres and Surface Temperatures. Science, 177: 52-56, July 1972. doi:10.1126/science.177.4043.52.

Schwabe, M. Sonnenbeobachtungen im Jahre 1843. Von Herrn Hofrath Schwabe in Dessau. Astronomische Nachrichten, 21: 233, Feb. 1844.

Skumanich, A. Time Scales for CA II Emission Decay, Rotational Braking, and Lithium Depletion. ApJ, 171: 565, Feb. 1972. doi:10.1086/151310.

Solanki, S. K., Krivova, N. A., \& Haigh, J. D. Solar Irradiance Variability and Climate. ARA\&A, 51: 311-351, Aug. 2013. doi:10.1146/annurev-astro-082812-141007.

Stocker, T., Qin, D., Plattner, G., Tignor, M., Allen, S., Boschung, J., Nauels, A., Xia, Y., Bex, 
B., \& Midgley, B. Ipcc, 2013: climate change 2013: the physical science basis. contribution of working group i to the fifth assessment report of the intergovernmental panel on climate change. 2013.

Willson, R. C. \& Hudson, H. S. The sun's luminosity over a complete solar cycle. Nature, 351: 42-44, May 1991. doi:10.1038/351042a0.

Wilson, O. C. Flux Measurements at the Centers of Stellar H- and K-Lines. ApJ, 153: 221, July 1968. doi:10.1086/149652.

Wilson, O. C. Chromospheric variations in main-sequence stars. ApJ, 226: 379-396, Dec. 1978. doi:10.1086/156618.

Yeo, K. L., Krivova, N. A., \& Solanki, S. K. Solar Cycle Variation in Solar Irradiance. Space Sci. Rev., 186: 137-167, Dec. 2014. doi:10.1007/s11214-014-0061-7. 